\title{
A Critical Analysis of the Mathematical Relationships and Comprehensiveness of Life Cycle Impact Assessment Approaches
}

\section{Jane C. Bare ${ }^{1}$, and Thomas P. Gloria ${ }^{2}$}

${ }^{1}$ U.S. Environmental Protection Agency, National Risk Management Research Laboratory, Cincinnati, Ohio 45268, USA. Phone: 513-569-7513. FAX: 513-569-7111. Email: bare.jane@epa.gov

2 ICF Consulting, 33 Hayden Avenue, Lexington, Massachusetts 02421, USA.

Email: tgloria@icfconsulting.com

\begin{abstract}
The impact assessment phase of Life Cycle Assessment (LCA) has received much criticism due to lack of consistency. While the ISO standards for LCA did make great strides in advancing the consensus in this area, ISO is not prescriptive, but has left much room for innovation and therefore inconsistency. To address this lack of consistency, there is currently an effort underway to provide a conceptual framework for Life Cycle Impact Assessment (LCIA) and recommended practice to include a list of impact categories, category indicators, and underlying methodologies. This is an enormous undertaking, especially in light of the current fundamental lack of consensus of the basic elements to be included in a LCIA (e.g., impact categories, impacts, and Areas of Protection). ISO 14042 requires selection of impact categories that "reflect a comprehensive set of environmental issues" related to the system being studied, especially for "comparative assertions" that involve public marketing claims. In order to be comprehensive it is necessary to have a listing of impacts that "could" be included within the LCIA before entering into discussions of impacts that "should" be included. In addition to providing a critical review of existing and emerging impact assessment approaches, this paper will formulate a structured representation that allows more informed selection of approaches. The definitions and relationships between midpoint, endpoint, damage, and Areas of Protection will be presented in greater detail, along with the equations that are common to many of the approaches. Finally, a discussion of the advantages and disadvantages of displaying results at various stages in the environmental models will be presented in great detail.
\end{abstract}

\section{Supporting Information}

This paper includes a detailed structure based on four Areas of Protection with four comprehensive tables (corresponding to each Area of Protection) allowing a comparison of eight popular impact assessment approaches. 


\begin{tabular}{|c|c|c|c|c|c|c|c|c|}
\hline \multicolumn{9}{|c|}{ Human Health } \\
\hline Method & CML & Eco-Indicator 99 & Ecoscarcity & EDIP97 & EPS 2000d & Impact 2002+ & LIME & TRACI \\
\hline \multicolumn{9}{|c|}{ Climate Protection } \\
\hline Midpoint & GWPs & Not reported separately. & GWPs & GWPs & Not reported separately. & GWP & GWPs & GWPs \\
\hline Endpoint & $\begin{array}{l}\text { All included. Not calculated } \\
\text { independently. }\end{array}$ & $\begin{array}{l}\text { Malaria, dengue fever, } \\
\text { schistosomiasis, cardio \& resp } \\
\text { disease, displaced populations }\end{array}$ & $\begin{array}{l}\text { All included. Not calculated } \\
\text { lindependently. }\end{array}$ & $\begin{array}{l}\text { All included. Not calculated } \\
\text { independently. }\end{array}$ & $\begin{array}{l}\text { Life expectancy, severe } \\
\text { morbidity, morbidity, severe } \\
\text { nuisance, nuisance }\end{array}$ & $\begin{array}{l}\text { Mortality and morbidity of } \\
\text { species }\end{array}$ & $\begin{array}{l}\text { Thermal stress, cold stress, } \\
\text { malaria, dengue fever, } \\
\text { disaster, food shortage }\end{array}$ & $\begin{array}{l}\text { All included. Not calculated } \\
\text { independently. }\end{array}$ \\
\hline Damage & Not calculated & DALYS & Not calculated & Not calculated & Person-years & Not calculated & DALYS & Not calculated \\
\hline Weighting & Not calculated & DALYs and 3 perspectives & Critical Flows & Political Reduction of Targets. & ELU/inidcator based on WTP & Not calculated & $\begin{array}{l}\text { (Yen/DALYs) or (WTP Yen * } \\
\text { Weighting Factor) }\end{array}$ & Not calculated \\
\hline \multicolumn{9}{|c|}{ Stratospheric Ozone Protection } \\
\hline Midpoint & ODPs & Not reported separately. & ODPs & ODPs & Not reported separately. & ODPs & ODPs & ODPs \\
\hline Endpoint & $\begin{array}{l}\text { All included. Not calculated } \\
\text { independently. }\end{array}$ & Skin cancers and cataracts. & $\begin{array}{l}\text { All included. Not calculated } \\
\text { independently. }\end{array}$ & $\begin{array}{l}\text { All included. Not calculated } \\
\text { independently. }\end{array}$ & Life expectancy & Cancer. & Unknown. & $\begin{array}{l}\begin{array}{l}\text { All included. Not calculated } \\
\text { independently. }\end{array} \\
\end{array}$ \\
\hline Damage & Not calculated & DALYS & Not calculated & Not calculated & Person-years & DALYS & DALYS & Not calculated \\
\hline Weighting & Not calculated & DALYs and 3 perspectives & Critical Flows & Political Reduction of Targets. & ELU/inidcator based on WTP & Not calculated & $\begin{array}{l}\text { (Yen/DALYs) or (WTP Yen* } \\
\text { Weighting Factor) }\end{array}$ & Not calculated \\
\hline \multicolumn{9}{|l|}{ Acidification } \\
\hline Midpoint & APs & None & APs & APs & Not reported separately. & None & None & APs \\
\hline Endpoint & $\begin{array}{l}\text { All included. Not calculated } \\
\text { independently. }\end{array}$ & None & $\begin{array}{l}\text { All included. Not calculated } \\
\text { independently. }\end{array}$ & $\begin{array}{l}\text { All included. Not calculated } \\
\text { independently. }\end{array}$ & $\begin{array}{l}\text { Life expectancy, severe } \\
\text { morbidity, morbidity, severe } \\
\text { nuisance, nuisance }\end{array}$ & None & None & $\begin{array}{l}\begin{array}{l}\text { All included. Not calculated } \\
\text { independently. }\end{array} \\
\text { ind }\end{array}$ \\
\hline Damage & Not calculated & None & Not calculated & Not calculated & Person-years & None & None & Not calculated \\
\hline Weighting & Not calculated & None & Critical Flows & Political Reduction of Targets. & ELU/inidcator based on WTP & None & None & Not calculated \\
\hline \multicolumn{9}{|c|}{ Smog Formation } \\
\hline Midpoint & PCOPs & Not reported separately. & Emissions maxima to air & PCOPs & Not reported separately. & PCOPs & PCOPs & PCOPs \\
\hline Endpoint & $\begin{array}{l}\text { All included. Not calculated } \\
\text { independently. }\end{array}$ & Not reported. & Not calculated & $\begin{array}{l}\text { All included. Not calculated } \\
\text { independently. }\end{array}$ & $\begin{array}{l}\text { Life expectancy, severe } \\
\text { morbidity, morbidity, severe } \\
\text { nuisance, nuisance }\end{array}$ & Respiratory & $\begin{array}{l}\text { Acute death, respiratory } \\
\text { disease, asthma attack }\end{array}$ & $\begin{array}{l}\begin{array}{l}\text { All included. Not calculated } \\
\text { independently. }\end{array} \\
\text { ind }\end{array}$ \\
\hline Damage & Not calculated & DALYS & Not calculated & Not calculated & Person-years & DALYS & DALYS & Not calculated \\
\hline Weighting & Not calculated & DALYS \& 3 perspectives & Critical Flows & Political Reduction of Targets. & ELU/inidcator based on WTP & Not calculated & $\begin{array}{l}\text { (Yen/DALYs) or (WTP Yen* } \\
\text { Weighting Factor) }\end{array}$ & Not calculated \\
\hline \multicolumn{9}{|c|}{ Eutrophication } \\
\hline Midpoint & Eutrophication Potentials & None & $\begin{array}{l}\text { Annual emissions: P:lakes; N: } \\
\text { saltwater basins }\end{array}$ & $\begin{array}{l}\text { N\&P equivalents based on } \\
\text { Redfield factor }\end{array}$ & Not reported separately. & None & None & Eutrophication Potentials \\
\hline Endpoint & $\begin{array}{l}\text { All included. Not calculated } \\
\text { independently. }\end{array}$ & None & Not calculated & $\begin{array}{l}\text { All included. Not calculated } \\
\text { independently. }\end{array}$ & $\begin{array}{l}\text { morbidity, morbidity, severe } \\
\text { nuisance, nuisance }\end{array}$ & None & None & $\begin{array}{l}\begin{array}{l}\text { All included. Not calculated } \\
\text { independently. }\end{array} \\
\end{array}$ \\
\hline Damage & Not calculated & None & Not calculated & Not calculated & Person-years & None & None & Not calculated \\
\hline Weighting & Not calculated & None & Critical Flows & Political Reduction of Targets. & ELU/inidcator based on WTP & None & None & Not calculated \\
\hline \multicolumn{9}{|l|}{ Cancer } \\
\hline Midpoint & $\begin{array}{l}\text { May be in other human toxicity } \\
\text { category. }\end{array}$ & Not calculated & $\begin{array}{l}\text { May be in other human toxicity } \\
\text { category. }\end{array}$ & None & Not reported separately. & $\begin{array}{l}\text { May be in other human toxicity } \\
\text { category. }\end{array}$ & $\begin{array}{l}\text { May be in other human toxicity } \\
\text { category. }\end{array}$ & Not calculated \\
\hline Endpoint & See above & Cancer & See above & None & $\begin{array}{l}\text { Life expectancy, severe } \\
\text { morbidity, morbidity, severe } \\
\text { nuisance, nuisance }\end{array}$ & See above & See above & Cancer \\
\hline Damage & See above & DALYS & See above & None & Person-years & See above & See above & HTPs \\
\hline Weighting & See above & DALYs \& 3 perspectives & See above & None & ELU/inidcator based on WTP & See above & See above & Not calculated \\
\hline \multicolumn{9}{|l|}{ Radiation } \\
\hline Midpoint & Radiation Effects & Not calculated & Not calculated & None & None & Not calculated & None & None \\
\hline Endpoint & Radiation Effects & Radiation Effects & Radiation Effects & None & None & Cancer. & None & None \\
\hline Damage & Yr kBq & DALYS & Volume of radioactive wastes & None & None & DALYS & None & None \\
\hline Weighting & Not calculated & DALYS \& 3 perspectives & Critical Flows & None & None & Not calculated & None & None \\
\hline \multicolumn{9}{|l|}{\begin{tabular}{|l} 
Respiratory \\
\end{tabular}} \\
\hline Midpoint & $\begin{array}{l}\text { May be in other human toxicity } \\
\text { category. }\end{array}$ & $\begin{array}{l}\text { May be found in Smog } \\
\text { Formation category. }\end{array}$ & $\begin{array}{l}\text { May be in other human toxicity } \\
\text { category. }\end{array}$ & $\begin{array}{l}\text { May be found in smog } \\
\text { formation category. }\end{array}$ & Not reported separately. & Not calculated & Not calculated & Not calculated \\
\hline Endpoint & See above & See above & See above & See above & \begin{tabular}{|l} 
Life expectancy, severe \\
morbidity, morbidity, severe \\
nuisance, nuisance
\end{tabular} & Respiratory & Respiratory disease. & Respiratory \\
\hline Damage & See above & See above & See above & See above & Person-years & DALYS & DALYS & HTPS \\
\hline Weighting & See above & See above & See above & See above & ELU/inidcator based on WTP & Not calculated & $\begin{array}{l}\text { (Yen/DALYs) or (WTP Yen* } \\
\text { Weighting Factor) }\end{array}$ & Not calculated \\
\hline \multicolumn{9}{|c|}{ Other Human Toxicity } \\
\hline Midpoint & Not calculated & None & Not calculated & Not calculated & Not reported separately. & Not calculated & Not calculated & Not calculated \\
\hline Endpoint & Range of toxicity effects tested & None & Range of toxicity effects & Range of toxicity effects & $\begin{array}{l}\text { Range of toxicity effects due to } \\
\text { metals (e.g., Hg) Life } \\
\text { expectancy, severe morbidity, } \\
\text { morbidity, severe nuisance }\end{array}$ & Range of toxicity effects & Respiratory disease \& cancer. & Noncancerous effects \\
\hline Damage & HTPS & None & $\begin{array}{l}\text { Emissions maxima to air, } \\
\text { water, and top soil }\end{array}$ & Not calculated & Person-years & DALYS & DALYS & Not calculated \\
\hline Weighting & Not calculated & None & Critical Flows & Political Reduction of Targets. & Political Reducation of Targets. & Not calculated & $\begin{array}{l}\text { (Yen/DALYYs) or (WTP Yen * } \\
\text { Weighting Factor) }\end{array}$ & Not calculated \\
\hline \multicolumn{9}{|l|}{ Odor } \\
\hline Midpoint & Not calculated & None & None & None & None & None & None & None \\
\hline Endpoint & Not calculated & None & None & None & Nuisance & None & None & None \\
\hline Damage & Malodorous air/water & None & None & None & Person-years & None & None & None \\
\hline Weighting & Not calculated & None & None & None & ELU/inidcator based on WTP & None & None & None \\
\hline \multicolumn{9}{|l|}{ Noise } \\
\hline Midpoint & $\begin{array}{l}\text { Unweighted aggregation of } \\
\text { sound }\end{array}$ & None & None & None & Not reported separately. & None & None & None \\
\hline Endpoint & Not calculated & None & None & None & Severe nuisance, nuisance & None & None & None \\
\hline Damage & Not calculated & None & None & None & Person-years & None & None & None \\
\hline Weighting & Not calculated & None & None & None & ELU/inidcator based on WTP & None & None & None \\
\hline \multicolumn{9}{|l|}{ Casualties } \\
\hline Midpoint & $\begin{array}{l}\text { Unweighted aggregation of } \\
\text { victims. }\end{array}$ & None & None & None & None & Not calculated & None & None \\
\hline Endpoint & Casualties & None & None & None & None & Casualties & None & None \\
\hline Damage & Not calculated & None & None & None & None & DALYS & None & None \\
\hline Weighting & Not calculated & None & None & None & None & None & None & None \\
\hline
\end{tabular}

Table 1: Comparing Human Health Modeling For Eight Impact Assessment Approaches 


\begin{tabular}{|c|c|c|c|c|c|c|c|c|}
\hline \multicolumn{9}{|c|}{ Natural Environmental Quality } \\
\hline Method & CML & Eco-Indicator 99 & Ecoscarcity & EDIP97 & EPS 2000d & Impact 2002+ & LIME & TRACI \\
\hline \multicolumn{9}{|c|}{ Climate Protection } \\
\hline Midpoint & GWPs & None. & GWPs & GWPs & Not reported separately. & GWPs & GWPs & GWPs \\
\hline Endpoint & $\begin{array}{l}\begin{array}{l}\text { All included. Not calculated } \\
\text { independently. }\end{array} \\
\end{array}$ & None. & $\begin{array}{l}\text { All included. Not calculated } \\
\text { independently. }\end{array}$ & $\begin{array}{l}\text { All included. Not calculated } \\
\text { independently. }\end{array}$ & $\begin{array}{l}\text { Ecosystem production capacity - } \\
\text { crop, wood, fish, meat, base cat- } \\
\text { ion capacity of soil, water }\end{array}$ & $\begin{array}{l}\text { Mortality and morbidity of } \\
\text { species }\end{array}$ & Terrestrial plant species & $\begin{array}{l}\begin{array}{l}\text { All included. Not calculated } \\
\text { independently. }\end{array} \\
\text { lint }\end{array}$ \\
\hline Damage & Not calculated. & None. & Not calculated. & Not calculated. & $\mathrm{kg}$ & Not calculated. & Yen/NPP dry ton or WTP (Yen) & Not calculated. \\
\hline Weighting & Not calculated. & None. & Critical Flows & Political Reduction of Targets. & ELU/indicator unit & Not calculated. & Yen/NPP dry ton or WTP (Yen) & Not calculated. \\
\hline \multicolumn{9}{|c|}{ Stratospheric Ozone Protection } \\
\hline Midpoint & ODPs & None. & JODPs & JoDPs & Not reported separately. & None. & ODPs & ODPs \\
\hline Endpoint & $\begin{array}{l}\text { All included. Not calculated } \\
\text { independently. }\end{array}$ & None. & $\begin{array}{l}\text { All included. Not calculated } \\
\text { independently. }\end{array}$ & $\begin{array}{l}\begin{array}{l}\text { All included. Not calculated } \\
\text { independently. }\end{array} \\
\end{array}$ & $\begin{array}{l}\text { Ecosystem production capacity - } \\
\text { crop, wood, fish, meat, base cat- } \\
\text { ion capacity of soil, water }\end{array}$ & None. & Forest and phytoplankton & $\begin{array}{l}\text { All included. Not calculated } \\
\text { independently. }\end{array}$ \\
\hline Damage & Not calculated. & None. & Not calculated. & Not calculated. & $\mathrm{kg}$ & None. & Yen/NPP dry ton or WTP (Yen) & Not calculated. \\
\hline Weighting & Not calculated. & None. & Critical Flows & Political Reduction of Targets. & ELU/indicator unit & None. & Yen/NPP dry ton or WTP (Yen) & Not calculated. \\
\hline \multicolumn{9}{|l|}{\begin{tabular}{|l|} 
Acidification \\
\end{tabular}} \\
\hline Midpoint & Acidification Potentials & Not calculated. & Acidification Potentials & Acidification Potentials & Not reported separately. & $\begin{array}{c}\text { errestrial and Aquatic } \\
\text { Acidification Potentials }\end{array}$ & None. & Acidification Potentials. \\
\hline Endpoint & $\begin{array}{l}\begin{array}{l}\text { All included. Not calculated } \\
\text { independently. }\end{array} \\
\end{array}$ & $\begin{array}{l}\text { Changed PH and Nutrient } \\
\text { Availability }\end{array}$ & $\begin{array}{l}\text { All included. Not calculated } \\
\text { independently. }\end{array}$ & $\begin{array}{l}\begin{array}{l}\text { All included. Not calculated } \\
\text { independently. }\end{array} \\
\end{array}$ & $\begin{array}{l}\text { Ecosystem production capacity - } \\
\text { crop, wood, fish, meat, base cat- } \\
\text { ion capacity of soil, water }\end{array}$ & $\begin{array}{l}\text { All included. Not calculated } \\
\text { independently. }\end{array}$ & None. & $\begin{array}{l}\begin{array}{l}\text { All included. Not calculated } \\
\text { independently. }\end{array} \\
\end{array}$ \\
\hline Damage & Not calculated. & $\begin{array}{l}\text { Potentrally UISappealed Frat } \\
\text { of Species }\end{array}$ & Not calculated. & Not calculated. & mol $\mathrm{H}+$ equivalents & Not calculated. & None. & Not calculated. \\
\hline Weighting & Not calculated. & PDFs \& 3 Perspectives & Critical Flows & Political Reduction of Targets. & ELU/indicator unit & Not calculated. & None. & Not calculated. \\
\hline \multicolumn{9}{|c|}{ Smog Formation } \\
\hline Midpoint & Smog Potentials & None. & Emissions maxima to air & Smog Potentials & None. & Smog Potentials & None. & Smog Potentials \\
\hline Endpoint & $\begin{array}{l}\text { All meluedeanoro } \\
\text { independently. }\end{array}$ & None. & Not calculated. & $\begin{array}{l}\text { Aitncludued. vor } \\
\text { independently. }\end{array}$ & None. & 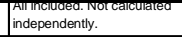 & None. & 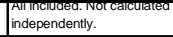 \\
\hline Damage & Not calculated. & None. & Not calculated. & Not calculated. & None. & Not calculated. & None. & Not calculated. \\
\hline Weighting & Not calculated. & None. & Critical Flows & Political Reduction of Targets. & None. & Not calculated. & None. & Not calculated. \\
\hline \multicolumn{9}{|c|}{ Eutrophication } \\
\hline Midpoint & Eutrophication Potentials & See above. & $\begin{array}{l}\text { Annual emissions:PIlakes; N: } \\
\text { saltwater basins }\end{array}$ & $\begin{array}{l}\text { N\&e equavalenis based on } \\
\text { Redfield factor }\end{array}$ & Not reported separately. & Eutrophication Potentials & None. & Eutrophication Potentials \\
\hline Endpoint & $\begin{array}{l}\text { All included. Not calculated } \\
\text { independently. }\end{array}$ & See above. & Not calculated. & $\begin{array}{l}\text { All included. Not calculated } \\
\text { (independently. }\end{array}$ & $\begin{array}{l}\text { Ecosystem production capacity - } \\
\text { crop, wood, fish, meat, base cat- } \\
\text { ion capacity of soil, water }\end{array}$ & $\begin{array}{l}\text { Mortality and morbidity of } \\
\text { species }\end{array}$ & None. & $\begin{array}{l}\text { All included. Not calculated } \\
\text { independently. }\end{array}$ \\
\hline Damage & Not calculated. & See above. & Not calculated. & Not calculated. & $\mathrm{kg}$ & Not calculated. & None. & Not calculated. \\
\hline Weighting & Not calculated. & See above. & Critical Flows & Political Reduction of Targets. & ELU/indicator unit & Not calculated. & None. & Not calculated. \\
\hline \multicolumn{9}{|l|}{ Radiation } \\
\hline \begin{tabular}{|l|} 
Midpoint \\
\end{tabular} & Radiation Effects & None. & Radiation Effects & None. & None. & None. & None. & None. \\
\hline Endpoint & Radiation Effects & None. & Radiation Effects & None. & None. & None. & None. & None. \\
\hline Damage & YrkBq & None. & Volume of radioactive wastes & None. & None. & None. & None. & None. \\
\hline Weighting & Not calculated. & None. & Critical Flows & None. & None. & None. & None. & None. \\
\hline \multicolumn{9}{|l|}{ Noise } \\
\hline Midpoint & $\begin{array}{l}\text { Unwergnted a } \\
\text { sound }\end{array}$ & None. & None. & None. & None. & None. & None. & None. \\
\hline Endpoint & Not calculated. & \begin{tabular}{|l} 
None. \\
\end{tabular} & None. & None. & None. & None. & None. & None. \\
\hline Damage & Not calculated. & None. & None. & None. & None. & \begin{tabular}{|l} 
None. \\
\end{tabular} & None. & None. \\
\hline Weighting & Not calculated. & None. & None. & None. & None. & \begin{tabular}{|l} 
None. \\
\end{tabular} & None. & None. \\
\hline
\end{tabular}

Table 2: Comparing Natural Environmental Quality For Eight Impact Assessment Approaches 


\begin{tabular}{|c|c|c|c|c|c|c|c|c|}
\hline \multicolumn{9}{|c|}{ Natural Resources } \\
\hline Method & $\overline{C M L}$ & Eco-Indicator 99 & Ecoscarcity & EDIP97 & EPS 2000d & Impact 2002+ & LIME & TRACI \\
\hline \multicolumn{9}{|c|}{ Fossil Fuel Depletion } \\
\hline Midpoint & None. & None. & None & None & None & None & None. & None \\
\hline Endpoint & $\begin{array}{l}\text { Energy carriers \& mineral } \\
\text { together. }\end{array}$ & Depletion of fossil fuel stock & $\begin{array}{l}\text { Fossil fuel use by energy } \\
\text { content }\end{array}$ & None & Depletion of fossil fuel stock & Depletion of fossil fuel stock & Total energy consumption & Depletion of fossil fuel stock \\
\hline Damage & $\begin{array}{l}\text { ADP in } \mathrm{kg} \text { antimony or } \mathrm{MJ} / \mathrm{kg} \\
\text { based on exergy content. }\end{array}$ & MJ surplus energy & Energy Use & None & $\mathrm{kg} / \mathrm{kg}$ reserves & $\begin{array}{l}\text { MJ primary non-renewable } \\
\text { energy }\end{array}$ & $\begin{array}{l}\text { The socio-economic impact } \\
\text { and impact on ecosystem }\end{array}$ & MJ surplus energy \\
\hline Weighting & None. & $\begin{array}{l}\text { MJ surplus energy \& } 3 \\
\text { perspectives }\end{array}$ & Critical Flows & None. & $\begin{array}{l}\text { ELU/ndicator unit based on } \\
\text { WTP }\end{array}$ & None & $\begin{array}{l}\text { Yen/NPP or WTP(Yen)* } \\
\text { Weighting Factor }\end{array}$ & None. \\
\hline \multicolumn{9}{|l|}{ Mineral Use } \\
\hline Midpoint & None. & Concentration of minerals & None & None & None & None & None. & None \\
\hline Endpoint & None. & Depletion of mineral stock & None & None & Depletion of mineral stock & Depletion of mineral stock & Inverse of resource reserve & None \\
\hline Damage & None. & MJ surplus energy & None & $\begin{array}{l}\text { Weighting based on supply } \\
\text { horizon }\end{array}$ & $\mathrm{kg} / \mathrm{kg}$ reserves & $\begin{array}{l}\text { MJ primary non-renewable } \\
\text { energy }\end{array}$ & $\begin{array}{l}\text { The socio-economic impact } \\
\text { and impact on ecosystem }\end{array}$ & None \\
\hline Weighting & None. & $\begin{array}{l}\text { MJ surplus energy \& } 3 \\
\text { perspectives }\end{array}$ & None & $\begin{array}{l}\text { Welghting based on supply } \\
\text { horizon }\end{array}$ & $\begin{array}{l}\text { ELU/rindicator unit based on } \\
\text { WTP }\end{array}$ & None & $\begin{array}{l}\text { Pen/IPP or WTP(Yen) } \\
\text { Weighting Factor }\end{array}$ & None \\
\hline \multicolumn{9}{|l|}{ Land Use } \\
\hline Midpoint & $\begin{array}{l}\text { Land competition \& loss of } \\
\text { biodiversity \& life support } \\
\text { function }\end{array}$ & $\begin{array}{l}\text { Change in habitat size and } \\
\text { land use }\end{array}$ & $\begin{array}{l}\text { Volume and weight of } \\
\text { controlled waste deposition } \\
\text { (use of scarce space) }\end{array}$ & None & Arable and forestry land & $\begin{array}{l}\text { Change in habitat size and } \\
\text { land use }\end{array}$ & $\begin{array}{l}\text { Land transformation and land } \\
\text { occupation }\end{array}$ & None \\
\hline Endpoint & Acres Utilized & $\begin{array}{l}\text { Mortality and morbiatity of } \\
\text { species (PDFs) }\end{array}$ & Waste deposition on land & None & $\begin{array}{l}\text { Morrallty and morbiatity of } \\
\text { species }\end{array}$ & $\begin{array}{l}\text { Morrallty and morbiatly or } \\
\text { species (PDFs) }\end{array}$ & $\begin{array}{l}\text { Measure of land occupled and } \\
\text { transformed }\end{array}$ & None \\
\hline Damage & Unweighted Acres & $\begin{array}{l}\text { Potentially Disappeared } \\
\text { Fraction of species (PDFs) }\end{array}$ & Waste deposition on land & None & $\begin{array}{l}\text { Normalized Extinction of } \\
\text { Species }\end{array}$ & \begin{tabular}{|l} 
Potentially Disappeared \\
Fraction of species (PDFs)
\end{tabular} & Vascular plant species & None \\
\hline Weighting & Not calculated. & PDFs \& 3 perspectives & Critical Flows & None & $\begin{array}{l}\text { ELU/indicator unit based on } \\
\text { WTP }\end{array}$ & None & $\begin{array}{l}\text { Yen/NPP or WTIP(Yen)' } \\
\text { Weighting Factor }\end{array}$ & None \\
\hline \multicolumn{9}{|l|}{ Water Use } \\
\hline Midpoint & Not specified. & None. & None & None & None & None & None. & None \\
\hline Endpoint & Not specified. & None. & None & None & None & None & None. & None \\
\hline Damage & Not specified. & None. & None & $\begin{array}{l}\text { Weighting based on supply } \\
\text { horizon }\end{array}$ & None & None & None. & None \\
\hline Weighting & Not specified. & None. & None & $\begin{array}{l}\text { Weighting based on supply } \\
\text { horizon }\end{array}$ & None & None & None. & None \\
\hline \multicolumn{9}{|c|}{ Other Resource Consumption } \\
\hline Midpoint & None. & J None. & None & None & None & None & None. & None \\
\hline Endpoint & None. & None. & None & None & None & None & \begin{tabular}{|l|} 
Total energy consumption \\
\end{tabular} & None \\
\hline Damage & None. & None. & None & $\begin{array}{l}\text { Weighting based on supply } \\
\text { horizon }\end{array}$ & None & None & $\begin{array}{l}\text { The socio-economic impact } \\
\text { and impact on ecosystem }\end{array}$ & None \\
\hline Weighting & None. & None. & None & $\begin{array}{l}\text { Weighting based on supply } \\
\text { horizon }\end{array}$ & None & None & $\begin{array}{l}\text { Yen/IPP or WTIP(YenT) } \\
\text { Weighting Factor }\end{array}$ & None \\
\hline \multicolumn{9}{|l|}{ Waste } \\
\hline Midpoint & None. & None. & See land use & None & None & None & See land use & See land use \\
\hline Endpoint & None. & None. & See land use & None & None & None & See land use & See land use \\
\hline Damage & None. & None. & See land use & None & None & None & See land use & See land use \\
\hline Weighting & None. & None. & See land use & None & None & None & See land use & See land use \\
\hline
\end{tabular}

Table 3: Comparing Natural Resource Modeling For Eight Impact Assessment Approaches

\begin{tabular}{|c|c|c|c|c|c|c|c|c|}
\hline \multicolumn{9}{|c|}{ Manmade Environment } \\
\hline Method & CML & Eco-Indicator 99 & Ecoscarcity & EDIP97/EDIP 2003 & EPS 2000d & Impact 2002+ & LIME & TRACI \\
\hline \multicolumn{9}{|l|}{ Abiotic } \\
\hline Midpoint & None & None & None & None & None & None & None & None \\
\hline Endpoint & None & None & None & None & \begin{tabular}{|l}
$\begin{array}{l}\text { Recreational and cultural } \\
\text { values }\end{array}$ \\
\end{tabular} & None & None & None \\
\hline Damage & None & None & None & None & None & None & \begin{tabular}{|l|} 
None \\
\end{tabular} & None \\
\hline Weighting & None & None & None & None & None & None & None & None \\
\hline \multicolumn{9}{|l|}{\begin{tabular}{|l} 
Biotic \\
\end{tabular}} \\
\hline \begin{tabular}{|l|} 
Midpoint \\
\end{tabular} & None & None & None & None & None & None & None & None \\
\hline Endpoint & None & None & None & None & None & None & None & None \\
\hline Damage & None & None & None & None & None & None & None & None \\
\hline \begin{tabular}{|l} 
Weighting \\
\end{tabular} & None & None & None & None & None & None & None & None \\
\hline
\end{tabular}

Table 4: Comparing Manmade Environment Modeling For Eight Impact Assessment Approaches 